\title{
Bergman completeness of Zalcman type domains
}

\author{
by \\ Piotr Jucha (Kraków)
}

\begin{abstract}
We give an equivalent condition for Bergman completeness of Zalcman type domains. This also solves a problem stated by Pflug.

The main subject of this paper is a class of planar domains - the so-called Zalcman type domains. We give an equivalent condition for the Bergman completeness of a wide class of such domains. This answers a question raised in [10]. Moreover, this gives a rich collection of domains which are Bergman complete but not Bergman exhaustive, i.e. which are counterexamples to Kobayashi's conjecture (see [7]).

To begin with, let us recall some necessary notions and properties connected with potential theory in the complex plane (see e.g. [12]).

Let $\mathcal{P}(K)$ be the set of all probability Borel measures $\mu$ with supports in a compact set $K \subset \mathbb{C}$. We define the logarithmic potential of $\mu \in \mathcal{P}(K)$ by

$$
p_{\mu}(z):=\int_{K} \log |z-w| d \mu(w), \quad z \in \mathbb{C} .
$$
\end{abstract}

A measure $\nu \in \mathcal{P}(K)$ is called the equilibrium measure of the set $K$ if

$$
I(\nu)=\sup \{I(\mu): \mu \in \mathcal{P}(K)\},
$$

where $I(\mu):=\int_{K} p_{\mu}(z) d \mu(z)$ is the energy of $\mu$. The logarithmic capacity of a set $E \subset \mathbb{C}$ is the number

$\operatorname{cap} E:=\exp (\sup \{I(\mu): \mu \in \mathcal{P}(K), K$ is a compact subset of $E\})$.

For a compact set $K \subset \mathbb{C}$ and $\zeta \in \mathbb{C} \backslash K$, let

$$
f_{K}(\zeta):= \begin{cases}\int_{K} \frac{d \mu_{K}(\lambda)}{\zeta-\lambda} & \text { if } \operatorname{cap} K>0 \\ 0 & \text { if } \operatorname{cap} K=0\end{cases}
$$

where $\mu_{K}$ denotes the equilibrium measure of $K$.

2000 Mathematics Subject Classification: Primary 32F45; Secondary 32A25, 30C40, $30 \mathrm{C} 85$. 
We set $\Delta\left(z_{0}, r\right):=\left\{z \in \mathbb{C}:\left|z-z_{0}\right|<r\right\}$ for $z_{0} \in \mathbb{C}, r>0$.

We will need the following properties:

(1) If $E_{1} \subset E_{2} \subset \mathbb{C}$, then cap $E_{1} \leq \operatorname{cap} E_{2}$.

(2) If $B=\bigcup_{k=1}^{N} B_{k}$, where $B_{k}$ are Borel sets in $\mathbb{C}$ and $\operatorname{diam} B \leq d$ $(d>0, N=1,2, \ldots, \infty)$, then

$$
\frac{1}{\log \left(\frac{d}{\operatorname{cap} B}\right)} \leq \sum_{k=1}^{N} \frac{1}{\log \left(\frac{d}{\operatorname{cap} B_{k}}\right)} .
$$

(3) $\operatorname{cap} \Delta(z, r)=\operatorname{cap} \partial \Delta(z, r)=r$.

(4) (Frostman's Theorem) Let $\mu$ be the equilibrium measure of a compact set $K$ such that cap $K>0$. Then $p_{\mu} \geq \log \operatorname{cap} K$ on $\mathbb{C}$ and $p_{\mu}=$ $\log$ cap $K$ on $K \backslash F$, where $F$ is an $F_{\sigma}$-subset of $\partial K$ such that cap $F$ $=0$. Moreover, if $z \in \partial K$ is regular for the Dirichlet problem for the unbounded connected component of $\mathbb{C} \backslash K$, then $p_{\mu}(z)=\log \operatorname{cap} K$.

(Wiener's criterion) Let $D \subset \mathbb{C}$ be a bounded domain and let $z_{0} \in$ $\partial D$. Fix $\theta \in(0,1)$. Define $F_{k}:=\left\{z \in \mathbb{C} \backslash D: \theta^{k+1} \leq\left|z-z_{0}\right|<\theta^{k}\right\}$. Then $z_{0}$ is a regular point for the Dirichlet problem for $D$ if and only if $\sum_{k=1}^{\infty}-k / \log \operatorname{cap} F_{k}=\infty$.

Let $L_{\mathrm{h}}^{2}(D)$ be the Hilbert space of square integrable functions holomorphic on $D \subset \mathbb{C}^{n}$ with the standard scalar product induced from $L^{2}(D)$ and norm $\|\cdot\|_{D}$. We define the Bergman kernel $K_{D}$ and the function $M_{D}$ for a bounded domain $D$ by

$$
\begin{aligned}
K_{D}(z) & :=\sup \left\{\frac{|f(z)|^{2}}{\|f\|_{D}^{2}}: f \in L_{\mathrm{h}}^{2}(D), f \not \equiv 0\right\}, \\
M_{D}(z ; X) & :=\sup \left\{\frac{\left|f^{\prime}(z) X\right|^{2}}{\|f\|_{D}^{2}}: f \in L_{\mathrm{h}}^{2}(D), f \not \equiv 0, f(z)=0\right\}
\end{aligned}
$$

for $z \in D$ and $X \in \mathbb{C}^{n}$. The Bergman metric $\beta_{D}$ is given by the formula

$$
\beta_{D}^{2}(z ; X):=\sum_{j, k=1}^{n} \frac{\partial^{2} \log K_{D}(z)}{\partial z_{j} \partial \bar{z}_{k}} X_{j} \bar{X}_{k}, \quad z \in D, X \in \mathbb{C}^{n},
$$

and the Bergman distance of $z, w \in D$ is

$b_{D}(z, w):=\inf \left\{L_{\beta_{D}}(\alpha) \mid \alpha:[0,1] \rightarrow D\right.$ piecewise $\left.\mathcal{C}^{1}, \alpha(0)=z, \alpha(1)=w\right\}$, where $L_{\beta_{D}}(\alpha):=\int_{0}^{1} \beta_{D}\left(\alpha(t), \alpha^{\prime}(t)\right) d t$.

If $D$ is a planar domain, then $M_{D}(z ; X)=|X|^{2} M_{D}(z ; 1)$ and $\beta_{D}(z ; X)=$ $|X| \beta_{D}(z ; 1)$. For simplicity, we write $M_{D}(z):=M_{D}(z ; 1)$ and $\beta_{D}(z):=$ $\beta_{D}(z ; 1)$. Recall that

$$
\beta_{D}^{2}(z)=\frac{M_{D}(z)}{K_{D}(z)}, \quad z \in D
$$


Let us also define, for $D \subset \mathbb{C}$,

$$
\gamma_{D}(z):=\int_{0}^{1 / 4} \frac{d \delta}{\delta^{3}(-\log \operatorname{cap}(\bar{\Delta}(z, \delta) \backslash D))}, \quad z \in D .
$$

A bounded domain $D \subset \mathbb{C}^{n}$ is called Bergman exhaustive at a point $z_{0} \in \partial D$ if $\lim _{D \ni z \rightarrow z_{0}} K_{D}(z)=\infty$. We say that $D$ is Bergman exhaustive if it is Bergman exhaustive at each of its boundary points.

A bounded domain $D$ is said to be Bergman complete if any Cauchy sequence with respect to the Bergman distance (a Cauchy-Bergman sequence) is convergent in the standard topology to a point of $D$.

We refer the reader to other publications for more properties of the Bergman kernel and metric (see e.g. [5]) and the function $\gamma_{D}$ (see [14], [11]).

It is known that hyperconvexity implies both exhaustiveness (see [8]) and Bergman completeness (see [1] and [4]). But the converse is not true (see e.g. [2], [4]).

On the complex plane, if a domain is Bergman exhaustive, then it is also Bergman complete (see [3]). A classification of Bergman exhaustive planar domains is also known:

Theorem 1 (see [14]). Let $D$ be a bounded domain in $\mathbb{C}, z_{0} \in \partial D$. Then

$$
\lim _{D \ni z \rightarrow z_{0}} \gamma_{D}(z)=\infty
$$

if and only if $D$ is Bergman exhaustive at $z_{0}$.

Kobayashi [7] asked whether exhaustiveness is necessary for completeness. After a long period of uncertainty, the answer turned out to be negative (see $[13])$.

Let us now define a special type of plane domains - the so-called Zalcman type domains:

$$
D:=\Delta(0,1) \backslash\left(\bigcup_{k=1}^{\infty} \bar{\Delta}\left(x_{k}, r_{k}\right) \cup\{0\}\right),
$$

where $x_{k}>x_{k+1}>0, \lim _{k \rightarrow \infty} x_{k}=0, \bar{\Delta}\left(x_{k}, r_{k}\right) \subset \Delta(0,1)$ and $\bar{\Delta}\left(x_{k}, r_{k}\right) \cap$ $\bar{\Delta}\left(x_{l}, r_{l}\right)=\emptyset$ for $k \neq l$.

We also consider additional conditions for such domains:

$$
\begin{aligned}
& \exists \theta_{1} \in(0,1) \forall k \geq 1: \quad \theta_{1} \leq \frac{x_{k+1}}{x_{k}} ; \\
& \exists \theta_{2} \in\left(\theta_{1}, 1\right) \forall k \geq 1: \quad \frac{x_{k+1}}{x_{k}} \leq \theta_{2} .
\end{aligned}
$$

The following useful corollary follows from Theorem 1. 
Corollary 2. Let $D$ be a domain given by (8) and satisfying (9) and (10). Then $D$ is Bergman exhaustive if and only if

$$
\sum_{k=1}^{\infty} \frac{-1}{x_{k}^{2} \log r_{k}}=\infty
$$

We prove the following

Theorem 3. Let $D$ be a domain given by (8) and satisfying (9) and (10). Then the following are equivalent:

(i) $D$ is Bergman complete,

(ii) $\sum_{k=1}^{\infty} 1 /\left(x_{k} \sqrt{-\log r_{k}}\right)=\infty$.

The following problem was stated in [10]: which domains satisfying (8) with

$$
x_{k}:=1 / 2^{k}
$$

are Bergman complete? Theorem 3 gives an answer to that question.

Regarding the hyperconvexity and exhaustiveness of these domains, we know everything.

THEOREM 4. If $D$ is a domain given by (8) and satisfying (12), then:

(a) $D$ is hyperconvex if and only if

$$
\sum_{k=1}^{\infty} \frac{k}{-\log r_{k}}=\infty
$$

(b) $D$ is Bergman exhaustive if and only if

$$
\sum_{k=1}^{\infty} \frac{2^{2 k}}{-\log r_{k}}=\infty
$$

Theorem 4 together with Theorem 3 gives us a rich collection of domains which are Bergman complete but not hyperconvex and, furthermore, Bergman complete but not Bergman exhaustive (they are simpler than those in [13]).

Incidentally, as a by-product of Theorem 3, we obtain (cf. Corollary 5 in [11])

Corollary 5. Let $D$ be a planar domain given by (8).

(a) If $D$ satisfies (10) then

$$
\sum_{N=1}^{\infty} \frac{1}{x_{N}^{2} \sqrt{-\log r_{N}}}<\infty \Rightarrow \limsup _{0>x \rightarrow 0} \beta_{D}(x)<\infty .
$$


(b) If D satisfies (9) and (10) then

$$
\limsup _{0>x \rightarrow 0} \beta_{D}(x)<\infty \Rightarrow \limsup _{N \rightarrow \infty} \frac{1}{x_{N}^{2} \sqrt{-\log r_{N}}}<\infty .
$$

For the proofs, we need the following lemma which is a straightforward corollary of Lemma 2 in [11].

Lemma 6. Given a bounded domain $D \subset \mathbb{C}$ and a number $\alpha \in(0,1)$, there is a constant $C>0$ such that for any compact set $K \subset \bar{\Delta}(0, \alpha)$ with $K \cap D=\emptyset$,

$$
\left\|f_{K}\right\|_{D} \leq C \sqrt{-\log \operatorname{cap}(K)} .
$$

Proof of Corollary 2. Notice that (7) holds for any $z_{0} \in \partial D \backslash\{0\}$. By Theorem 1, we only need to prove that (11) is equivalent to $\lim _{D \ni z \rightarrow 0} \gamma_{D}(z)$ $=\infty$.

We have $\bar{\Delta}\left(x_{k+1}-\frac{1}{2} r_{k+1}, \frac{1}{2} r_{k+1}\right) \subset \bar{\Delta}(0, \delta) \backslash D$ for $\delta \in\left(x_{k+1}, x_{k}\right)$. Consequently, from the definition of $\gamma_{D}$ and using (9) and (10), we obtain

$$
\begin{aligned}
\gamma_{D}(0) & \geq \sum_{k=k_{0}}^{\infty} \int_{x_{k+1}}^{x_{k}} \frac{d \delta}{\delta^{3}(-\log \operatorname{cap}(\bar{\Delta}(0, \delta) \backslash D))} \geq \sum_{k=k_{0}}^{\infty} \int_{x_{k+1}}^{x_{k}} \frac{d \delta}{-\delta^{3} \log \frac{1}{2} r_{k+1}} \\
& \geq \sum_{k=k_{0}}^{\infty}\left(x_{k}-x_{k+1}\right) \frac{-1}{x_{k}^{3} \log \frac{1}{2} r_{k+1}} \geq C \sum_{k=k_{0}}^{\infty} \frac{-1}{x_{k+1}^{2} \log r_{k+1}} .
\end{aligned}
$$

Above, $k_{0}$ is an integer such that $x_{k_{0}}<1 / 4$ and $C$ is a numerical constant. Now, the divergence of the series in (11) implies that $\gamma_{D}(0)=\infty$. Due to the lower semicontinuity of $\gamma_{D}$ (see [14]) we deduce that $\lim _{D \ni z \rightarrow 0} \gamma_{D}(z)=\infty$.

On the other hand, we have

$$
\begin{aligned}
\gamma_{D}(0) & =\left(\int_{x_{1}}^{1 / 4}+\sum_{k=1}^{\infty} \int_{x_{k+1}}^{x_{k}}\right) \frac{d \delta}{\delta^{3}(-\log \operatorname{cap}(\bar{\Delta}(0, \delta) \backslash D))} \\
& \leq C_{1}+\sum_{k=1}^{\infty} \frac{x_{k}-x_{k+1}}{x_{k+1}^{3}} \sum_{j=k}^{\infty} \frac{1}{-\log r_{j}} \stackrel{(9)}{\leq} C_{1}+C_{2} \sum_{j=1}^{\infty} \sum_{k=1}^{j} \frac{1}{x_{k}^{2}} \frac{1}{-\log r_{j}} \\
& \stackrel{(10)}{\leq} C_{1}+C_{3} \sum_{j=1}^{\infty} \frac{-1}{x_{j}^{2} \log r_{j}} .
\end{aligned}
$$

Above, $C_{1} \geq 0$ and $C_{2}, C_{3}>0$ are constants. The last inequality holds due to $(10)$ :

$$
\sum_{k=1}^{j} \frac{1}{x_{k}^{2}} \leq \sum_{k=1}^{j} \frac{\theta_{2}^{2(j-k)}}{x_{j}^{2}}<\frac{1}{1-\theta_{2}^{2}} \frac{1}{x_{j}^{2}} .
$$

Thus, if the series in (11) is convergent, then $\gamma_{D}(0)<\infty$. We can deduce directly from the definition of $\gamma_{D}$ that $\gamma_{D}\left(y_{1}\right) \leq \gamma_{D}\left(y_{2}\right)$ for $-1 / 4 \leq y_{1} \leq$ 
$y_{2} \leq 0$. Hence, if $\gamma_{D}(0)<\infty$, then also $\lim \sup _{0>z \rightarrow 0} \gamma_{D}(z)<\infty$. This finishes the proof.

Proof of Theorem 3. (i) $\Rightarrow$ (ii) (cf. the proof of Theorem 3 in [11]). Suppose that

$$
\sum_{k=1}^{\infty} \frac{1}{x_{k} \sqrt{-\log r_{k}}}<\infty .
$$

It is sufficient to show that there exists a $\delta>0$ such that $\int_{-\delta}^{0} \beta_{D}(x) d x<\infty$.

Let us introduce some notations:

$$
\begin{aligned}
K_{0}:=\bar{\Delta}(0,1) \backslash \Delta\left(0,1-\varepsilon_{0}\right), \quad K_{j}:=\bar{\Delta}\left(x_{j}, r_{j}\right), \quad j \geq 1, \\
L_{j}:=\bigcup_{k=j+1}^{\infty} \bar{\Delta}\left(x_{k}, r_{k}\right) \cup \bar{\Delta}\left(0, \varepsilon_{j}\right), \quad j \geq 1, \\
\widetilde{D}_{j}:=\left(D \cup \Delta\left(0, \varepsilon_{j}\right)\right) \cap \Delta\left(0,1-\varepsilon_{0}\right),
\end{aligned}
$$

where $\varepsilon_{0}<1 / 4$ is fixed. We choose $\varepsilon_{j} \in\left(0, x_{j+1}\right)$ so small that

$$
\frac{1}{-\log \operatorname{cap} L_{j}}<2 \sum_{k=j+1}^{\infty} \frac{1}{-\log r_{k}}
$$

(apply (2) and (3)) and such that $\partial \Delta\left(0, \varepsilon_{j}\right) \subset D$. For a compact set $B \subset \mathbb{C}$, let $p_{B}:=p_{\mu_{B}}$ be the logarithmic potential. If cap $B>0$, we choose a function $\chi_{B} \in \mathcal{C}^{\infty}(\mathbb{R},[0,1])$ such that

$$
\chi_{B}(t)= \begin{cases}1 & \text { if } t \leq \log \operatorname{cap} B \\ 0 & \text { if } t \geq \frac{1}{2} \log \operatorname{cap} B\end{cases}
$$

and

$$
\left|\chi_{B}^{\prime}(t)\right| \leq \frac{4}{-\log \operatorname{cap} B}, \quad t \in \mathbb{R} .
$$

Let $\varphi_{B}:=\chi_{B} \circ p_{B}$. Then $\varphi_{B} \equiv 1$ on $B$ by Frostman's theorem. We will use the following lemma which will be proven later.

Lemma 7. Given a domain $D$ as in Theorem 3 satisfying (14), there exists an integer $N_{0}$ such that for $j \geq N_{0}$ :

$$
\begin{aligned}
& \operatorname{supp} \varphi_{K_{j}} \subset \bar{\Delta}\left(0,1-2 \varepsilon_{0}\right), \quad \operatorname{supp} \varphi_{L_{j}} \subset \bar{\Delta}\left(0,1-2 \varepsilon_{0}\right) ; \\
& \operatorname{supp} \varphi_{K_{j}} \cap \operatorname{supp} \varphi_{K_{j+1}}=\emptyset ; \\
& \operatorname{supp} \varphi_{K_{j}} \cap \operatorname{supp} \varphi_{L_{j}}=\emptyset ; \\
& \operatorname{dist}\left(-x_{j}, \operatorname{supp} \varphi_{L_{j}}\right) \geq \frac{1}{2}\left(1-\theta_{2}\right) x_{j} .
\end{aligned}
$$

The behavior of the Bergman kernel and metric is a local property (see e.g. Theorem 6.3.5 in [5]). So, without loss of generality, we may assume that (17)-(20) hold for all $j \geq 1$. 
Choose one more function $\varphi_{0} \in \mathcal{C}^{\infty}(\mathbb{R},[0,1])$ such that $\varphi_{0} \equiv 1$ on $K_{0}$ and $\operatorname{supp} \varphi_{0} \subset \bar{\Delta}\left(0,1+\varepsilon_{0}\right) \backslash \Delta\left(0,1-2 \varepsilon_{0}\right)$. Then, by (17), we also have $\operatorname{supp} \varphi_{0} \cap \bigcup_{j=1}^{\infty}\left(\operatorname{supp} \varphi_{K_{j}} \cup \operatorname{supp} \varphi_{L_{j}}\right)=\emptyset$.

Now,

$$
\left|\frac{\partial \varphi_{B}}{\partial \bar{z}}(z)\right|=\left|\chi_{B}^{\prime}\left(p_{B}(z)\right) \frac{\partial p_{B}(z)}{\partial \bar{z}}\right| \leq \frac{4\left|f_{B}(z)\right|}{-\log \operatorname{cap} B}, \quad z \in D,
$$

where $B=K_{j}$ or $B=L_{j}$ for $j \geq 1$, and

$$
\left|\frac{\partial \varphi_{0}}{\partial \bar{z}}(z)\right| \leq M, \quad z \in D
$$

where $M>0$ is a constant.

Now, take any $N \in \mathbb{N}$, choose $x \in\left[-x_{N-1},-x_{N}\right]$, and put

$$
\varphi:=\varphi_{0}+\varphi_{K_{1}}+\ldots+\varphi_{K_{N}}+\varphi_{L_{N}} .
$$

Then $\varphi \equiv 1$ on $\partial \widetilde{D}_{N}$.

Take any $f \in L_{\mathrm{h}}^{2}(D), f \not \equiv 0$. Using the Cauchy integral formula and the Green formula, we obtain

$$
\begin{aligned}
\left|f^{\prime}(x)\right|= & \frac{1}{2 \pi}\left|\int_{\partial \widetilde{D}_{N}} \frac{f(\lambda) d \lambda}{(\lambda-x)^{2}}\right|=\frac{1}{2 \pi}\left|\int_{\partial \widetilde{D}_{N}} \frac{f(\lambda) \varphi(\lambda) d \lambda}{(\lambda-x)^{2}}\right| \\
= & \frac{1}{\pi}\left|\int_{\widetilde{D}_{N}} \frac{f(\lambda)}{(\lambda-x)^{2}} \frac{\partial \varphi}{\partial \bar{\lambda}}(\lambda) d L^{2}(\lambda)\right| \\
\leq & \frac{1}{\pi} \int_{\widetilde{D}_{N}} \frac{|f(\lambda)|}{|\lambda-x|^{2}}\left|\frac{\partial \varphi_{0}}{\partial \bar{\lambda}}(\lambda)\right| d L^{2}(\lambda) \\
& +\sum_{j=1}^{N} \frac{1}{\pi} \int_{\widetilde{D}_{N}} \frac{|f(\lambda)|}{|\lambda-x|^{2}}\left|\frac{\partial \varphi_{K_{j}}}{\partial \bar{\lambda}}(\lambda)\right| d L^{2}(\lambda) \\
& +\frac{1}{\pi} \int_{\widetilde{D}_{N}} \frac{|f(\lambda)|}{|\lambda-x|^{2}}\left|\frac{\partial \varphi_{L_{N}}}{\partial \bar{\lambda}}(\lambda)\right| d L^{2}(\lambda) .
\end{aligned}
$$

Now, we use the Cauchy-Schwarz inequality and the estimates $1 /|\lambda-x| \leq$ $C_{1} / x_{j}$ for $\lambda \in \operatorname{supp} \varphi_{K_{j}}, j=1, \ldots, N$, and $1 /|\lambda-x| \leq C_{1} / x_{N}$ for $\lambda \in$ $\operatorname{supp} \varphi_{L_{N}}$ (due to $\left.(20)\right)$ :

$$
\left|f^{\prime}(x)\right| \leq C_{2}\|f\|_{D}\left(1+\sum_{j=1}^{N} \frac{1}{x_{j}^{2}} \frac{\left\|f_{K_{j}}\right\|_{D}}{-\log \operatorname{cap} K_{j}}+\frac{1}{x_{N}^{2}} \frac{\left\|f_{L_{N}}\right\|_{D}}{-\log \operatorname{cap} L_{N}}\right) .
$$


Finally, using Lemma 6, we obtain

$$
\left|f^{\prime}(x)\right| \leq C_{3}\|f\|_{D}\left(1+\sum_{j=1}^{N} \frac{1}{x_{j}^{2} \sqrt{-\log \operatorname{cap} K_{j}}}+\frac{1}{x_{N}^{2} \sqrt{-\log \text { cap } L_{N}}}\right) .
$$

The constants $C_{1}, C_{2}, C_{3}>0$ above do not depend on $N$.

Thus

$$
\sqrt{M_{D}(x)} \leq C_{3}\left(1+\sum_{j=1}^{N} \frac{1}{x_{j}^{2} \sqrt{-\log r_{j}}}+\frac{\sqrt{2}}{x_{N}^{2}} \sum_{j=N+1}^{\infty} \frac{1}{\sqrt{-\log r_{j}}}\right)
$$

owing to (15).

Now, let us move on to the final estimations:

$$
\begin{gathered}
\int_{-x_{1}}^{0} \sqrt{M_{D}(x)} d x=\sum_{N=2}^{\infty} \int_{-x_{N-1}}^{-x_{N}} \sqrt{M_{D}(x)} d x \\
\stackrel{(9)}{\leq} \sum_{N=2}^{\infty} C_{4} x_{N} \sup _{x \in\left[-x_{N-1},-x_{N}\right]} \sqrt{M_{D}(x)} \\
\leq C_{5}\left(\sum_{N=2}^{\infty} x_{N}+\sum_{N=2}^{\infty} x_{N} \sum_{j=1}^{N} \frac{1}{x_{j}^{2} \sqrt{-\log r_{j}}}+\sum_{N=2}^{\infty} \frac{1}{x_{N}} \sum_{j=N+1}^{\infty} \frac{1}{\sqrt{-\log r_{j}}}\right),
\end{gathered}
$$

where $C_{4}, C_{5}>0$ are some constants. The first series in brackets is finite because of (10). For the second series, we have

$$
\begin{aligned}
\sum_{N=1}^{\infty} x_{N} \sum_{j=1}^{N} \frac{1}{x_{j}^{2} \sqrt{-\log r_{j}}} & =\sum_{j=1}^{\infty}\left(\sum_{N=j}^{\infty} x_{N}\right) \frac{1}{x_{j}^{2} \sqrt{-\log r_{j}}} \\
& \leq \frac{1}{1-\theta_{2}} \sum_{j=1}^{\infty} \frac{1}{x_{j} \sqrt{-\log r_{j}}} \stackrel{(14)}{<} \infty
\end{aligned}
$$

because (10) implies $\sum_{N=j}^{\infty} x_{N} \leq \sum_{s=0}^{\infty} \theta_{2}^{s} x_{j}=x_{j} /\left(1-\theta_{2}\right)$. Notice also that

$$
\sum_{N=1}^{j-1} \frac{1}{x_{N}} \leq \sum_{s=1}^{j-1} \theta_{2}^{s} \frac{1}{x_{j}}<\frac{\theta_{2}}{1-\theta_{2}} \frac{1}{x_{j}} \text {. }
$$

Thus,

$$
\begin{aligned}
\sum_{N=1}^{\infty} \frac{1}{x_{N}} \sum_{j=N+1}^{\infty} \frac{1}{\sqrt{-\log r_{j}}} & =\sum_{j=2}^{\infty}\left(\sum_{N=1}^{j-1} \frac{1}{x_{N}}\right) \frac{1}{\sqrt{-\log r_{j}}} \\
& \leq \frac{\theta_{2}}{1-\theta_{2}} \sum_{j=1}^{\infty} \frac{1}{x_{j} \sqrt{-\log r_{j}}} \stackrel{(14)}{<} \infty
\end{aligned}
$$

As a consequence, $\int_{-x_{1}}^{0} \beta_{D}(x) d x<\infty$ because $K_{D}$ is separated from 0 on $D$. 
(ii) $\Rightarrow$ (i) (cf. the proof of Theorem 5 in [13]). Suppose that $D$ is Bergman exhaustive at 0 . Since $D$ is Bergman exhaustive at any other point, it is Bergman complete by the result of Chen (see [3]).

Thus, we can assume that $D$ is not Bergman exhaustive at 0 . In view of Corollary 2 , the series (11) is convergent. Hence, $\lim _{j \rightarrow \infty} 1 /\left(x_{j} \sqrt{-\log r_{j}}\right)$ $=0$.

We are going to use an auxiliary lemma which will be proven later.

Lemma 8. Let $D$ be a domain as in Theorem 3 with

$$
\sum_{j=1}^{\infty} \frac{1}{x_{j} \sqrt{-\log r_{j}}}=\infty \quad \text { and } \quad \lim _{j \rightarrow \infty} \frac{1}{x_{j} \sqrt{-\log r_{j}}}=0 .
$$

If $\gamma:[0,1) \rightarrow D$ is a curve such that $\lim _{t \rightarrow 1} \gamma(t)=0$ and $\left.\gamma\right|_{[0, t]}$ is piecewise $\mathcal{C}^{1}$ for all $t \in(0,1)$, then $\int_{\gamma} \sqrt{M_{D}(z)} d l(z)=\infty$.

Suppose that $D$ is not Bergman complete. Then there exists a CauchyBergman sequence $\left(z_{k}\right)_{k=1}^{\infty} \subset D$ such that $\lim _{k \rightarrow \infty} z_{k}=0$. We can choose the sequence such that $b_{D}\left(z_{k}, z_{k+1}\right)<1 / 2^{k+1}$. We join each pair of points $z_{k}, z_{k+1}$ by a $\mathcal{C}^{1}$-curve of $L_{\beta_{D}}$-length not greater than $1 / 2^{k}$. The curve which we obtain by gluing all the small pieces, say $\gamma:[0,1) \rightarrow D$, has a finite length with respect to the Bergman metric. We set $\gamma^{\star}:=\gamma([0,1))$.

Notice that the Bergman kernel $K_{D}$ must be bounded on $\gamma^{\star}$. In fact, suppose the opposite. Then there is a sequence $\left(w_{k}\right)_{k=1}^{\infty} \subset \gamma^{\star}$ such that $\lim _{k \rightarrow \infty} w_{k}=0$ and $\lim _{k \rightarrow \infty} K_{D}\left(w_{k}\right)=\infty$. This sequence is also a CauchyBergman sequence. Then, by the results of Pflug ([9]) and Chen (see [2], $[3])$, there is a subsequence $\left(w_{k_{j}}\right)_{j=1}^{\infty}$ and a function $f \in L_{\mathrm{h}}^{2}(D)$ such that

$$
\frac{\left|f\left(w_{k_{j}}\right)\right|}{\sqrt{K_{D}\left(w_{k_{j}}\right)}} \rightarrow 1, \quad j \rightarrow \infty .
$$

Because the functions from $L_{\mathrm{h}}^{2}(D)$ bounded in a neighborhood of 0 are dense in $L_{\mathrm{h}}^{2}(D)$ (see [3]), there exists a $g \in L_{\mathrm{h}}^{2}(D)$ such that $\|g-f\|_{D} \leq 1 / 2$ and $g$ is bounded near 0 . Thus, also by the general properties of the Bergman kernel $K_{D}$, we have

$$
\frac{\left|g\left(w_{k_{j}}\right)\right|}{\sqrt{K_{D}\left(w_{k_{j}}\right)}} \geq \frac{\left|f\left(w_{k_{j}}\right)\right|}{\sqrt{K_{D}\left(w_{k_{j}}\right)}}-\|f-g\|_{D} \geq \frac{\left|f\left(w_{k_{j}}\right)\right|}{\sqrt{K_{D}\left(w_{k_{j}}\right)}}-\frac{1}{2} .
$$

Letting $j \rightarrow \infty$ yields a contradiction and we conclude that $K_{D}$ is bounded on $\gamma^{\star}$.

Finally, we use Lemma 8, which leads to a contradiction:

$$
\infty>\int_{\gamma^{\star}} \beta_{D}(z) d l(z) \geq \frac{1}{\sup _{\gamma^{\star}} \sqrt{K_{D}}} \int_{\gamma^{\star}} \sqrt{M_{D}(z)} d l(z)=\infty .
$$

As a consequence, the domain $D$ is Bergman complete. 
Proof of Lemma \%. We see that $\operatorname{supp} \varphi_{B} \subset\left\{z \in \mathbb{C}: p_{B}(z) \leq \frac{1}{2} \log \operatorname{cap} B\right\} \subset\{z \in \mathbb{C}: \operatorname{dist}(z, B) \leq \sqrt{\operatorname{cap} B}\}$ for a compact set $B$ with cap $B>0$.

Let $\delta_{0}>0$ be small. For sufficiently large $j$ (say $j \geq N_{0}=N_{0}\left(\delta_{0}\right)$ ), we have

$$
\begin{aligned}
& \operatorname{cap} K_{j}=r_{j} \stackrel{(*)}{\leq} \delta_{0}^{2} x_{j}^{2}, \\
& \operatorname{cap} L_{j} \leq \frac{-1}{\log \operatorname{cap} L_{j}} \stackrel{(15)}{\leq} 2 \sum_{k=j+1}^{\infty} \frac{-1}{\log r_{k}} \stackrel{(*)}{\leq} 2 \delta_{0}^{2} x_{j+1}^{2} .
\end{aligned}
$$

Both the inequalities marked with $(*)$ hold since $\sum_{k=1}^{\infty}-1 /\left(x_{k}^{2} \log r_{k}\right)$ is convergent (by (14)). The latter inequality is true because

$$
\frac{1}{x_{j+1}^{2}} \sum_{k=j+1}^{\infty} \frac{-1}{\log r_{k}} \leq \sum_{k=j+1}^{\infty} \frac{-1}{x_{k}^{2} \log r_{k}} \leq \delta_{0}^{2}
$$

if $j \geq 1$ is large enough.

Thus, we have

$$
\begin{aligned}
& \operatorname{supp} \varphi_{K_{j}} \subset K_{j}+\bar{\Delta}\left(0, \delta_{0} x_{j}\right), \\
& \operatorname{supp} \varphi_{L_{j}} \subset L_{j}+\bar{\Delta}\left(0, \sqrt{2} \delta_{0} x_{j+1}\right), \quad j \geq N_{0} .
\end{aligned}
$$

Therefore, the conditions (17)-(20) are fulfilled provided that $\delta_{0}$ is chosen small enough and $N_{0}$ is sufficiently large. Indeed, (17) is straightforward whereas (18), (19) and (20) follow from the inequalities, respectively:

$$
\begin{aligned}
r_{j}+\delta_{0} x_{j}+r_{j+1}+\delta_{0} x_{j+1} & <x_{j}-x_{j+1}, \\
r_{j}+\delta_{0} x_{j}+r_{j+1}+\sqrt{2} \delta_{0} x_{j+1} & <x_{j}-x_{j+1}, \\
\varepsilon_{j}+\sqrt{2} \delta_{0} x_{j+1}+\frac{1}{2}\left(1-\theta_{2}\right) x_{j} & <x_{j} .
\end{aligned}
$$

The above inequalities can be obtained by the use of (10), lowering $\delta_{0}$ if necessary. Recall that we have chosen $\varepsilon_{j}<x_{j+1}$ and $r_{j} \leq \delta_{0}^{2} x_{j}^{2}$.

Proof of Lemma 8. Without loss of generality, we may assume that $|\gamma(0)|>x_{1}$ and

$$
x_{1} \sqrt{-\log r_{1}}<x_{j} \sqrt{-\log r_{j}}, \quad j>1 .
$$

Fix $N \geq 2$ and take $z_{0} \in D$ such that $x_{N+2} \leq\left|z_{0}\right| \leq x_{N+1}$. Define

$$
f:=f_{\bar{\Delta}\left(x_{1}, r_{1}\right)}-\frac{x_{N}-z_{0}}{x_{1}-z_{0}} f_{\bar{\Delta}\left(x_{N}, r_{N}\right)} .
$$

For a disk, we have the following formula:

$$
f_{\bar{\Delta}(x, r)}(z)=\frac{1}{2 \pi} \int_{0}^{2 \pi} \frac{d t}{z-x-r e^{i t}}=\frac{1}{x-z} .
$$


So, we can explicitly compute that

$$
f\left(z_{0}\right)=0, \quad f^{\prime}\left(z_{0}\right)=\frac{x_{N}-x_{1}}{\left(x_{1}-z_{0}\right)^{2}\left(x_{N}-z_{0}\right)} .
$$

Using Lemma 6, we obtain the estimate

$$
\begin{aligned}
&\|f\|_{D} \leq\left\|f_{\bar{\Delta}\left(x_{1}, r_{1}\right)}\right\|+\frac{\left|x_{N}-z_{0}\right|}{\left|x_{1}-z_{0}\right|}\left\|f_{\bar{\Delta}\left(x_{N}, r_{N}\right)}\right\| \\
& \leq C_{1}\left(\sqrt{-\log r_{1}}+\frac{\left|x_{N}-z_{0}\right|}{\left|x_{1}-z_{0}\right|} \sqrt{-\log r_{N}}\right) \\
& \stackrel{(10),(23)}{\leq} C_{2} \frac{\left|x_{N}-z_{0}\right|}{\left|x_{1}-z_{0}\right|} \sqrt{-\log r_{N}},
\end{aligned}
$$

where $C_{1}, C_{2}>0$ are constants independent of $N$. Hence,

$$
\begin{aligned}
\sqrt{M_{D}\left(z_{0}\right)} & \geq \frac{\left|f^{\prime}\left(z_{0}\right)\right|}{\|f\|_{D}} \geq \frac{x_{1}-x_{N}}{C_{2}\left|x_{1}-z_{0}\right|\left|x_{N}-z_{0}\right|^{2} \sqrt{-\log r_{N}}} \\
& \stackrel{(10)}{\geq} \frac{C_{3}}{x_{N}^{2} \sqrt{-\log r_{N}}},
\end{aligned}
$$

where $C_{3}>0$ is a constant. Finally,

$$
\begin{aligned}
\int_{\gamma} \sqrt{M_{D}(z)} d l(z) & \geq \sum_{N=2}^{\infty} \inf _{|z| \in\left[x_{N+2}, x_{N+1}\right]} \sqrt{M_{D}(z)}\left(x_{N+1}-x_{N+2}\right) \\
& \geq C_{3} \sum_{N=2}^{\infty} \frac{x_{N+1}-x_{N+2}}{x_{N}^{2} \sqrt{-\log r_{N}}} \\
& \stackrel{(9),(10)}{\geq} C_{4} \sum_{N=2}^{\infty} \frac{1}{x_{N} \sqrt{-\log r_{N}}}=\infty .
\end{aligned}
$$

This finishes the proof.

Proof of Theorem 4. (a) We know that hyperconvexity of a bounded domain is equivalent to the regularity of the Dirichlet problem (see e.g. $[6,12])$. Applying Wiener's criterion (5) to the point $z_{0}=0$, we also get equivalence to (13). Note that for $\theta=1 / 2$ in (5), due to the properties (1)-(3), we have

$$
\frac{1}{-\log \left(\frac{1}{2} r_{k}\right)} \leq \frac{1}{-\log \operatorname{cap} F_{k}} \leq \frac{1}{-\log r_{k}}+\frac{1}{-\log r_{k+1}}
$$

because

$$
\Delta\left(\frac{1}{2^{k}}-\frac{1}{2} r_{k}, \frac{1}{2} r_{k}\right) \subset F_{k} \subset \Delta\left(\frac{1}{2^{k+1}}, r_{k+1}\right) \cup \Delta\left(\frac{1}{2^{k}}, r_{k}\right) .
$$


Then also

$$
\frac{1}{2} \sum_{k=1}^{\infty} \frac{k}{-\log r_{k}} \leq \sum_{k=1}^{\infty} \frac{k}{-\log \operatorname{cap} F_{k}} \leq 2 \sum_{k=1}^{\infty} \frac{k}{-\log r_{k}} .
$$

(b) That is a consequence of Corollary 2 .

Proof of Corollary 5. (a) By (21), the convergence of the series $\sum_{N=1}^{\infty} 1 /\left(x_{N}^{2} \sqrt{-\log r_{N}}\right)$ implies that $\sqrt{M_{D}(x)}<C$ for all $x \in[-1 / 2,0)$ and some numerical constant $C>0$. Thus, the Bergman metric $\beta_{D}$ is also bounded on $[-1 / 2,0)$ because the Bergman kernel $K_{D}$ is separated from 0 on $D$. Notice that we do not use (9) in the proof of (21).

(b) Suppose that $\lim _{\sup _{0>x \rightarrow 0}} \beta_{D}(x)<\infty$. Then $K_{D}$ must be bounded on $[-1 / 2,0)$ and $(23)$ holds (reason as in the proof of Theorem 3 , second part). To complete the proof, use (24).

Acknowledgements. The author would like to thank Professor Włodzimierz Zwonek for fruitful discussions and useful hints, as well as for pointing out mistakes in earlier versions of the paper.

\section{References}

[1] Z. Błocki and P. Pflug, Hyperconvexity and Bergman completeness, Nagoya Math. J. 151 (1998), 221-225.

[2] B.-Y. Chen, Completeness of the Bergman metric on non-smooth pseudoconvex domains, Ann. Polon. Math. 71 (1999), 242-251.

[3] -, A remark on the Bergman completeness, Complex Variables Theory Appl. 42 (2000), no. 1, 11-15.

[4] G. Herbort, The Bergman metric on hyperconvex domains, Math. Z. 232 (1999), 183-196.

[5] M. Jarnicki and P. Pflug, Invariant Distances and Metrics in Complex Analysis, de Gruyter, Berlin, 1993.

[6] M. Klimek, Pluripotential Theory, Oxford Univ. Press, 1991.

[7] S. Kobayashi, Geometry of bounded domains, Trans. Amer. Math. Soc. 92 (1959), 267-290.

[8] T. Ohsawa, On the Bergman kernel of hyperconvex domains, Nagoya Math. J. 129 (1993), 43-52.

[9] P. Pflug, Various applications of the existence of well growing holomorphic functions, in: Functional Analysis, Holomorphy and Approximation Theory, J. A. Barossa (ed.), North-Holland Math. Stud. 71, North-Holland, 1982.

[10] -, Invariant metrics and completeness, J. Korean Math. Soc. 37 (2000), 269-284.

[11] P. Pflug and W. Zwonek, Logarithmic capacity and Bergman functions, Arch. Math. (Basel) 80 (2003), 536-552.

[12] T. Ransford, Potential Theory in the Complex Plane, Cambridge Univ. Press, 1995.

[13] W. Zwonek, An example concerning Bergman completeness, Nagoya Math. J. 164 (2001), 89-102. 
[14] W. Zwonek, Wiener's type criterion for Bergman exhaustiveness, Bull. Polish Acad. Sci. Math. 50 (2002), 297-311.

Institute of Mathematics

Jagiellonian University

Reymonta 4

30-059 Kraków, Poland

E-mail: jucha@im.uj.edu.pl

Received December 3, 2002

Revised version November 25, 2003 